\title{
PD-L1 expression in small cell lung cancer: should we designate it for assignment?
}

\author{
Taiki Hakozaki^ \\ Department of Thoracic Oncology and Respiratory Medicine, Tokyo Metropolitan Cancer and Infectious Diseases Center of Komagome Hospital, \\ Tokyo, Japan \\ Correspondence to: Taiki Hakozaki, MD. Department of Thoracic Oncology and Respiratory Medicine, Tokyo Metropolitan Cancer and Infectious \\ Diseases Center of Komagome Hospital, 3-18-22 Honkomagome, Tokyo 113-0021, Japan. Email: t-hakozaki@cick.jp. \\ Comment on: Lee YS, Lim JH, Ryu W, et al. The clinical impact of three validated PD-L1 immunohistochemistry assays as a prognostic factor in \\ small cell lung cancer. Transl Lung Cancer Res 2021;10:2539-50.
}

Submitted Sep 03, 2021. Accepted for publication Sep 18, 2021.

doi: $10.21037 /$ tlcr-21-720

View this article at: https://dx.doi.org/10.21037/tlcr-21-720

Immune checkpoint inhibitors (ICIs) have revolutionized the therapeutic landscape of patients with advanced cancers across their primary organs and histological subtypes. Small cell lung cancer (SCLC) is no exception. Pivotal phase III trials IMpower 133 (1), CASPIAN (2) demonstrated significant improvement of progression-free survival (PFS) and overall survival (OS) by adding anti-PD-L1 antibody (atezolizumab, durvalumab) to first-line platinum doublet chemotherapy for extensive stage disease SCLC (ED-SCLC). In late line settings, the United States Food and Drug Administration (FDA) granted accelerated approval for nivolumab monotherapy as a third-line treatment of ED-SCLC, based on subset analysis of the CheckMate 032 study (3). Thus, ICIs are surely integrated into the current standard of care $(\mathrm{SoC})$ for $\mathrm{ED}$ SCLC. Now, we thoracic oncologists need to debilitate their optimal utilization in clinical practice. However, it would be still just an ideal theory. With relatively modest improvement of PFS and OS by on the addition of ICI in overall patients with ED-SCLC, we have no idea who will benefit from this new modality at the individual level. Even if there is no malicious intent, we might put a portion of patients at risk of immune-related adverse events (irAEs) with scant clinical benefit. Financial toxicity cannot also be ignored. In other words, predictive biomarkers identifying patients with ED-SCLC for whom ICIs might offer a durable benefit (or unnecessary risk; irAEs) are crucially deficient.

We have already been experiencing similar situations for immunotherapy in patients with advanced non-small cell lung cancer (NSCLC), a few years in advance. Patient selection in NSCLC according to PD-L1 expression on tumor and/or immune cells (ICs) in the tumor microenvironment worked to a certain extent. Now, regimens in the combinations with ICIs and platinum doublet chemotherapy are SoC as first-line therapy for advanced NSCLC irrespective of PD-L1 expression. For PD-L 1 positive $(\geq 1 \%$ or $\geq 50 \%)$ patients, ICI monotherapy is a possible treatment option in first-line settings. In countries where both chemo-immunotherapy and ICI monotherapy regimens are available, clinicians can choose regimens considering their risk-benefit for individual patients based on the PD-L1 expression level. Thus, although PD-L1 expression cannot be a definitive biomarker for NSCLC, it can be utilized in clinical decision making to a certain extent. Given the situation, we may well expect to stratify the prognosis of patients with SCLC according to PD-L1 expression in the era of pan-cancer immunotherapy.

In an original article, Lee et al. (4) explored the prognostic impact of PD-L1 expression in 59 patients with SCLC, using archival tissue samples before clinical

^ ORCID: 0000-0002-9980-4417. 
application of immunotherapy for ED-SCLC. In their study, 47 patients $(80 \%)$ received active treatment beyond platinum doublet chemotherapy and 30 patients $(51 \%)$ had limited disease stage (LD-SCLC) at tissue sampling. The authors also compared three immunochemistry (IHC) assays in their associations with OS. Although the results seem not to be practice-changing, their research, in comparison with available data on the similar topic, presents us an opportunity to reconsider the current positioning of PD-L1 based approach for patients with SCLC.

First, we need to grasp the clinicopathological features, such as prevalence and prognostic impact of the PDL1 expression level in tissues from patients with SCLC separated from immunotherapy. As Lee et al. pointed out, despite a high mutation burden, the prevalence of PD-L1 expression in SCLC seems to be lower compared to that reported for NSCLC or other cancer types. In preceding studies in which the cutoff value for PD-L1 positivity evaluated by FDA approved IHC assays (28-8, 22C3, SP263 or SP142) set at $1 \%$ or $5 \%$, the prevalence of PD-L1 positivity ranged from $10-40 \%$ (4). For SCLC, clinical data focused on the scantiness of "high" $(\geq 50 \%)$ PD-L1 expression, probably due to its low prevalence of such a high expressor in this population. In the cohort of Lee et al., the PD-L1 expression of most samples ranged under 5\% and only a few exhibited positivity, with the maximum being at $30 \%$. As for the association between survival and PD-L1 positivity, preceding studies have not provided a definite conclusion $(5,6)$. In a recent metanalysis including nine clinical studies, no statistically significant associations were observed between OS and PD-L1 positivity defined either $\geq 1 \%$ or $\geq 5 \%$, pooled hazard ratio (HR) of all studies: $0.86,95 \%$ confidence interval (CI): 0.49-1.50, $\mathrm{P}=0.59$ (6). These results give way to the question of whether PD-L1 positivity set at such low cutoff can be a promising biomarker for SCLC. We might need to recognize that the PD-L1 axis may play a lesser role in the pathophysiology of SCLC compared to that in other tumors. Although future accumulation of data on PD-L1 high SCLC may provide more suggestions on this issue, the low prevalence would get in the way of the clinical applicability of the PD-L1 based approach. At least, we need to set clearer clinical goals in the exploration of this approach for SCLC: whether we would like to define patients with poor prognosis who need alternative or more intensive therapeutic strategies, or those with excellent prognosis on immunotherapy. To take a step forward, we would need more data on the prognostic impact of PD-L1 among SCLC, including "high expressor", as well as their distribution to grasp a whole picture. In addition, disease state (ED-SCLC or LD-SCLC) and treatment modalities will also need to be considered in future research, as the clinical significance of initial PD-L1 expression at diagnosis can differ between patients' clinical scenario. Preceding studies including the work by Lee et al. have not fully investigated this point.

Among the questions on PD-L1 expression in SCLC, clinicians' immediate interest would mainly lie in its applicability as a predictive biomarker for immunotherapy. Subset analyses from pivotal phase III trials provide us with some of its reputation in the context of chemoimmunotherapy for ED-SCLC. In the exploratory biomarker analysis from the IMpower 133 trial, PD-L1 evaluation was performed using an SP263 assay in 34\% $(n=137)$ of the intention-to-treat population $(n=403)(7)$. In the tumor microenvironment, PD-L1 expression was observed mostly on tumor-infiltrating ICs, with limited expression on tumor cells (TCs). The prevalence of PD-L1 expression (either on ICs or TCs) $\geq 1 \%$ was $53 \%$, and that of $\geq 5 \%$ was $21.2 \%$. In this updated survival analysis report, OS and PFS data according to PD-L1 expression was presented. In the subgroup of patients with PD-L1 expression $\geq 5 \% \quad(n=29)$, a better OS tendency was observed in the chemo-immunotherapy compared with the chemotherapy alone group (median OS: $21.6 \mathrm{vs}$. 9.2 months, HR: $0.60,95 \%$ CI: $0.25-1.46, \mathrm{P}=0.25)$. An OS benefit with chemo-immunotherapy was observed even in the subgroup of PD-L1 negative $(<1 \%$ both on TCs and ICs) patients $(\mathrm{n}=65)$ (median OS: $10.2 \mathrm{vs}$. 8.3 months, HR: 0.51, 95\% CI: 0.30-0.89, $\mathrm{P}=0.015$ ), as well as PFS benefit in this subgroup (median PFS: 5.4 vs. 4.2 months, HR: 0.52, 95\% CI: 0.31-0.88, $\mathrm{P}=0.014)$. Furthermore, Kaplan-Meier curves for OS were almost overlapped in the subgroup of patients with PD-L1 expression $\geq 1 \% \quad(\mathrm{n}=72)$ (median OS: 9.7 vs. 10.6 months, HR: 0.87, 95\% CI: 0.51-1.49, $\mathrm{P}=0.61)$. Similar overlapped curves were observed in PFS analysis for PD-L1 expression $\geq 1 \%$ subgroup (median PFS: 5.1 vs. 5.5 months, HR: 0.86, 95\% CI: 0.51-1.46, $\mathrm{P}=0.58$ ). Thus, the PD-L1-based approach failed to stratify patients' prognosis, both in terms of OS and PFS, on chemo-immunotherapy. A similar survival benefit of chemo-immunotherapy over placebo plus platinum doublet was observed across PD-L1 subgroups in the exploratory analysis in CASPIAN (8) and KEYNOTE-604 (9). Although these are not confirmatory results from exploratory analysis with a relatively small sample size 
in each PD-L1 subgroup, we would need to admit that non-positive signals on the applicability PD-L1 status as a predictive biomarker for chemo-immunotherapy were presented with reproducibility. Here again, we seem to be encouraged to explore alternative practical approaches for the personalization of immunotherapy, at least chemo-immunotherapy, for patients with SCLC.

The discordances of PD-L1 positivity (on TCs or ICs) among the IHC assays are causing another confusion. For lung cancer, the feasibility of harmonizing these assays was assessed by an international panel of pathologist in the blueprint project (BP). In phase 1 of the $\mathrm{BP}(\mathrm{n}=38)$ in which tissues of NSCLC were stained with four validated IHC assays (28-8, 22C3, SP263 and SP142), a lower sensitivity of the SP142 assay for detecting PD-L1 expression on TCs, as well as the interchangeability of the 28-8, 22C3 and SP263 assays was observed (10). Phase 2 of the $\mathrm{BP}(\mathrm{n}=81)$, which included 71 NSCLC and 10 SCLC samples, consolidated the results from phase 1 the study (11). Consistent with preceding studies, the proportion of PD-L1 positive samples among SCLC was low; only 1 of the 10 SCLC samples showed staining for PD-L1. Although we still have scant data on the interchangeability of these IHC assays in PD-L1 evaluation for SCLC, it would be wise to recognize the lower sensitivity of the SP142 assays in the detection of PD-L1 expression, especially on TCs. In their study with 59 SCLC samples, Lee et al. showed the associations between PD-L1 expression of the SP142 assay, along with that of at least 1 of the 3 IHC assays (22C3, SP263 and SP142) and longer OS. Although the methodology of their analysis was validated, caution is still warranted in the interpretation of their results. Patients with "relatively high" PD-L1 expression in their cohort, not detected by the other IHC assays, may exhibit better prognosis than those with "allnegative" PD-L1 expression. In addition, the distribution of PD-L1 expression in the tumor microenvironment (i.e., TCs or ICs) was not taken into account. Depending on the point of view, the strength of their study may rather lie in providing hints for the interchangeability of different IHC assays for SCLC, with a larger cohort $(n=59)$ than preceding reports. As Lee et al. pointed out, validation with larger cohorts in harmonization of the IHC assays is needed to judge the clinical applicability of the PD-L1 approach for SCLC.

It would not still be practical for thoracic oncologists to integrate the PD-L1 based approach into clinical decision making for patients with ED-SCLC, as we did for advanced NSCLC. Although we recently got immunotherapy as a new treatment option for SCLC, the clinicopathological features of SCLC seem to make its personalization not going straightforward. These tough situations would suggest that there is still a strong need for exploring alternative treatment options and biomarkers for SCLC, along with optimizations of existing modalities.

\section{Acknowledgments}

Funding: None.

\section{Footnote}

Provenance and Peer Review: This article was commissioned by the editorial office, Translational Lung Cancer Research. The article did not undergo external peer review.

Conflicts of Interest: The author has completed the ICMJE uniform disclosure form (available at https://dx.doi. org/10.21037/tlcr-21-720). Taiki Hakozaki has received personal fees from Chugai Pharmaceutical outside the submitted work.

Ethical Statement: The author is accountable for all aspects of the work in ensuring that questions related to the accuracy or integrity of any part of the work are appropriately investigated and resolved.

Open Access Statement: This is an Open Access article distributed in accordance with the Creative Commons Attribution-NonCommercial-NoDerivs 4.0 International License (CC BY-NC-ND 4.0), which permits the noncommercial replication and distribution of the article with the strict proviso that no changes or edits are made and the original work is properly cited (including links to both the formal publication through the relevant DOI and the license). See: https://creativecommons.org/licenses/by-nc-nd/4.0/.

\section{References}

1. Horn L, Mansfield AS, Szczęsna A, et al. First-Line Atezolizumab plus Chemotherapy in Extensive-Stage Small-Cell Lung Cancer. N Engl J Med 2018;379:2220-9.

2. Paz-Ares L, Dvorkin M, Chen Y, et al. Durvalumab plus platinum-etoposide versus platinum-etoposide in firstline treatment of extensive-stage small-cell lung cancer (CASPIAN): a randomised, controlled, open-label, phase 3 trial. Lancet 2019;394:1929-39. 
3. Ready N, Farago AF, de Braud F, et al. Third-Line Nivolumab Monotherapy in Recurrent SCLC: CheckMate 032. J Thorac Oncol 2019;14:237-44.

4. Lee YS, Lim JH, Ryu W, et al. The clinical impact of three validated PD-L1 immunohistochemistry assays as a prognostic factor in small cell lung cancer. Transl Lung Cancer Res 2021;10:2539-50.

5. Cai H, Zhang H, Jiang Y. Prognostic and Clinicopathological Value of Programmed Cell Death Ligand1 Expression in Patients With Small Cell Lung Cancer: A Meta-Analysis. Front Oncol 2020;10:1079.

6. Acheampong E, Abed A, Morici M, et al. Tumour PDL1 Expression in Small-Cell Lung Cancer: A Systematic Review and Meta-Analysis. Cells 2020;9:2393.

7. Liu SV, Reck M, Mansfield AS, et al. Updated Overall Survival and PD-L1 Subgroup Analysis of Patients With Extensive-Stage Small-Cell Lung Cancer Treated With Atezolizumab, Carboplatin, and Etoposide (IMpower133). J Clin Oncol 2021;39:619-30.

Cite this article as: Hakozaki T. PD-L1 expression in small cell lung cancer: should we designate it for assignment? Transl Lung Cancer Res 2021;10(9):3697-3700. doi: 10.21037/tlcr21-720
8. Paz-Ares L, Goldman JW, Garassino MC, et al. PD-L1 expression, patterns of progression and patient-reported outcomes (PROs) with durvalumab plus platinumetoposide in ES-SCLC: Results from CASPIAN. Ann Oncol 2019;30:v851-v934.

9. Rudin CM, Awad MM, Navarro A, et al. Pembrolizumab or Placebo Plus Etoposide and Platinum as First-Line Therapy for Extensive-Stage Small-Cell Lung Cancer: Randomized, Double-Blind, Phase III KEYNOTE-604 Study. J Clin Oncol 2020;38:2369-79.

10. Hirsch FR, McElhinny A, Stanforth D, et al. PDL1 Immunohistochemistry Assays for Lung Cancer: Results from Phase 1 of the Blueprint PD-L1 IHC Assay Comparison Project. J Thorac Oncol 2017;12:208-22.

11. Tsao MS, Kerr KM, Kockx M, et al. PD-L1 Immunohistochemistry Comparability Study in Real-Life Clinical Samples: Results of Blueprint Phase 2 Project. J Thorac Oncol 2018;13:1302-11. 